\title{
Extensive variation in synonymous substitution rates in mitochondrial genes of seed plants Jeffrey P Mower ${ }^{1,3}$, Pascal Touzet ${ }^{2}$, Julie S Gummow ${ }^{1}$, Lynda F Delph ${ }^{1}$ and Jeffrey D Palmer*1
}

Address: ${ }^{1}$ Department of Biology, Indiana University, Bloomington, IN, 47405, USA, ${ }^{2}$ Laboratoire de Genetique et Evolution des Populations Vegetales, UMR CNRS 8016, Universite des Sciences et Technologies de Lille - Lille1, France and ${ }^{3}$ Smurfit Institute of Genetics, Trinity College Dublin, Dublin 2, Ireland

Email: Jeffrey P Mower - mowerj@tcd.ie; Pascal Touzet - pascal.touzet@univ-lille1.fr; Julie S Gummow - jsgummow@gmail.com; Lynda F Delph - ldelph@indiana.edu; Jeffrey D Palmer* - jpalmer@indiana.edu

* Corresponding author

Published: 9 August 2007

BMC Evolutionary Biology 2007, 7:135 doi:10.1/86/147|-2/48-7-135
Received: 15 April 2007

Accepted: 9 August 2007

This article is available from: http://www.biomedcentral.com/I47/-2/48/7//35

(C) 2007 Mower et al; licensee BioMed Central Ltd.

This is an Open Access article distributed under the terms of the Creative Commons Attribution License (http://creativecommons.org/licenses/by/2.0), which permits unrestricted use, distribution, and reproduction in any medium, provided the original work is properly cited.

\begin{abstract}
Background: It has long been known that rates of synonymous substitutions are unusually low in mitochondrial genes of flowering and other land plants. Although two dramatic exceptions to this pattern have recently been reported, it is unclear how often major increases in substitution rates occur during plant mitochondrial evolution and what the overall magnitude of substitution rate variation is across plants.
\end{abstract}

Results: A broad survey was undertaken to evaluate synonymous substitution rates in mitochondrial genes of angiosperms and gymnosperms. Although most taxa conform to the generality that plant mitochondrial sequences evolve slowly, additional cases of highly accelerated rates were found. We explore in detail one of these new cases, within the genus Silene. A roughly 100 -fold increase in synonymous substitution rate is estimated to have taken place within the last 5 million years and involves only one of ten species of Silene sampled in this study. Examples of unusually slow sequence evolution were also identified. Comparison of the fastest and slowest lineages shows that synonymous substitution rates vary by four orders of magnitude across seed plants. In other words, some plant mitochondrial lineages accumulate more synonymous change in 10,000 years than do others in 100 million years. Several perplexing cases of gene-to-gene variation in sequence divergence within a plant were uncovered. Some of these probably reflect interesting biological phenomena, such as horizontal gene transfer, mitochondrial-to-nucleus transfer, and intragenomic variation in mitochondrial substitution rates, whereas others are likely the result of various kinds of errors.

Conclusion: The extremes of synonymous substitution rates measured here constitute by far the largest known range of rate variation for any group of organisms. These results highlight the utility of examining absolute substitution rates in a phylogenetic context rather than by traditional pairwise methods. Why substitution rates are generally so low in plant mitochondrial genomes yet occasionally increase dramatically remains mysterious. 


\section{Background}

A synonymous site substitution is defined as a change in a protein-coding gene that does not alter the amino acid sequence encoded by the gene. Thus, synonymous substitutions are often assumed to be free of selection at the protein level, and the rates at which they accumulate are widely used as an approximation of the neutral mutation rate. The first study on rates of synonymous substitutions in flowering plants found that mitochondrial genes evolve a few times more slowly than chloroplast genes, about ten times more slowly than plant and mammal nuclear genes, and 50-100 times more slowly than mammalian mitochondrial genes [1]. Later studies have confirmed the low rate of synonymous changes in angiosperm mitochondrial genes [2-4] and extended the observation to the entire mitochondrial genome [5]. However, recent studies identified two genera of flowering plants, Plantago and Pelargonium, that have experienced a dramatic increase in the mitochondrial rate of synonymous substitution [6-8]. Some of these rate increases were temporary, with rates approaching or returning to normally low levels in certain descendent lineages $[6,7]$.

Phylogenetic analyses have suggested additional cases of rate acceleration for several plant lineages [9-14], but it is unclear whether this is a widespread phenomenon in plants. Here we test the generality of slow synonymous sequence evolution in plant mitochondrial genes across a large number (between 306 and 578 species, depending on the gene) and wide diversity of seed plants (i.e., gymnosperms and angiosperms). Although genes from most species evolve slowly, as expected, additional cases of highly accelerated rates were found, as were examples of exceptionally slow sequence evolution. Surprisingly, a few plants were also identified that contain a mixture of both quickly and slowly evolving mitochondrial genes. Overall, these results demonstrate that the synonymous substitution rate in plant mitochondria is a more variable character than previously appreciated.

\section{Results \\ Levels of sequence divergence in mitochondrial genes of seed plants}

Previous studies identified two plant lineages, Plantago and the Geraniaceae (especially Pelargonium), that have independently experienced periods of accelerated substitution rates in their mitochondrial genes [6-8]. To determine whether additional cases of rate acceleration could be found in mitochondrial genes of other seed plants (gymnosperms and angiosperms), blast searches were undertaken in order to collect all available atp1, cox1, and $m a t R$ homologues from GenBank. These three genes were chosen because they have been used in a number of broad phylogenetic studies and their sequences are available for hundreds of different species of plants. After removing multiple sequences from the same species, pseudogenes, cDNAs, and short gene fragments, a total of 546, 306, and 578 sequences were available for atp1, $\operatorname{cox} 1$, and matR, respectively. For each gene, homologous sequences were aligned and maximum likelihood (ML) analyses were performed using the Muse-Gaut codon model [15] to obtain estimates of synonymous site divergence $\left(d_{\mathrm{S}}\right)$ per branch (Figs. 1, 2, 3).

A surprising feature of these trees is the amount of branchlength variation seen (Figs. 1, 2, 3). Although the great majority of plants show conventionally low levels of synonymous site divergence, a number of species have substantially shorter or longer branches for one or more genes. The most striking examples of increased sequence divergence are from Plantago and Pelargonium, whose atp 1 and cox 1 branch lengths are exceptionally long (note that the matR gene has not been isolated from Pelargonium or from the fastest members of Plantago), as previously reported $[6,7]$. Although less extreme, one or more species from Silene, Apodanthes, Acorus, Ephedra, and Podocarpus are also very divergent across multiple genes. Patterns are not as clear for other taxa. For instance, Goodenia and Musa are highly divergent for atp1 but only moderately divergent for cox1. For a number of species, such as Carex and Anthericum, a sequence from one gene is very divergent but data are not available for the other two genes.

In contrast to the examples of increased levels of mitochondrial sequence divergence, there are several groups that consistently show an unusually low level of divergence, even in the context of the general conservation expected for plants (Figs. 1, 2, 3). Within the angiosperms, these groups include the Arecales (also see [16]), the Chloranthales, the sister orders Laurales and Magnoliales, and most orders within the basal eudicots. Several gymnosperm lineages also have short branches, including the Cycadales, the Pinaceae, and Ginkgo.

Unexpectedly, these analyses have also identified several lineages with high levels of divergence for some genes but low levels for others (Figs. 1, 2, 3). For example, divergence levels for Alisma are high for atp1 and matR but unexceptional for $\operatorname{cox} 1$. The $\operatorname{cox} 1$ and matR genes from Ranunculus are remarkable in that they are unusually conserved, similar to the very slow rates found in most basal eudicots, while its atp1 gene is moderately divergent. For Polemonium and Pentamerista (in the Tetrameristaceae), atp1 is divergent but matR is conserved. Conversely, matR is divergent but atp1 is conserved for Pilostyles (in the Apodanthaceae).

To more accurately assess levels of sequence divergence across seed plants, synonymous $\left(d_{\mathrm{S}}\right)$ and nonsynonymous 


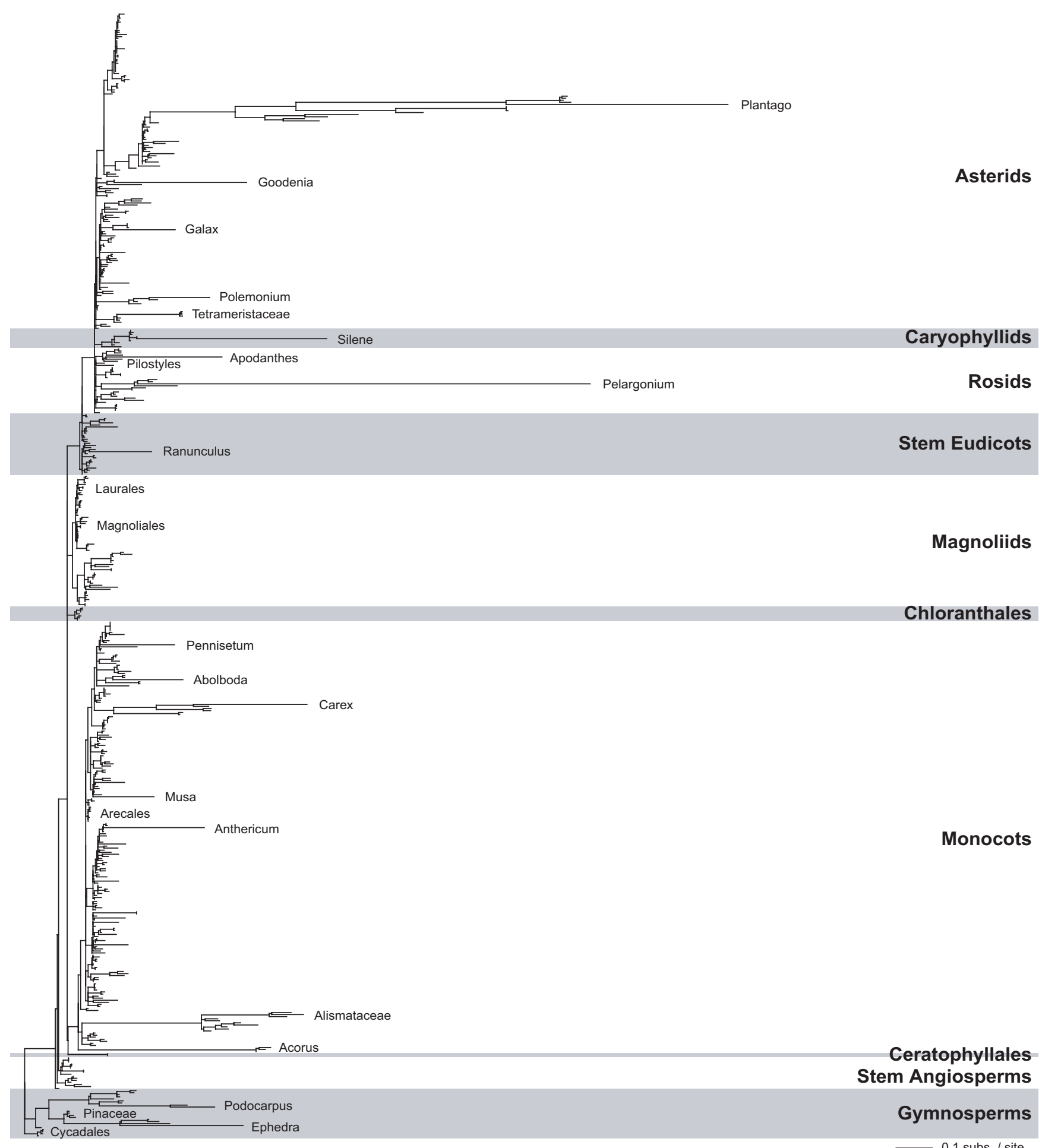

\section{Figure I}

Synonymous sequence divergence for mitochondrial atpl. Shown is the $d_{S}$ tree resulting from a codon-based likelihood analysis of the mitochondrial atp / gene. Branch lengths are in units of synonymous substitutions per synonymous site. All unique species available in GenBank (546) were included in the analysis. Topological constraints were enforced during the analysis (see methods). Names are shown only for taxa with unusually long or short branches. Figures I, 2, and 3 are drawn to the same scale. 


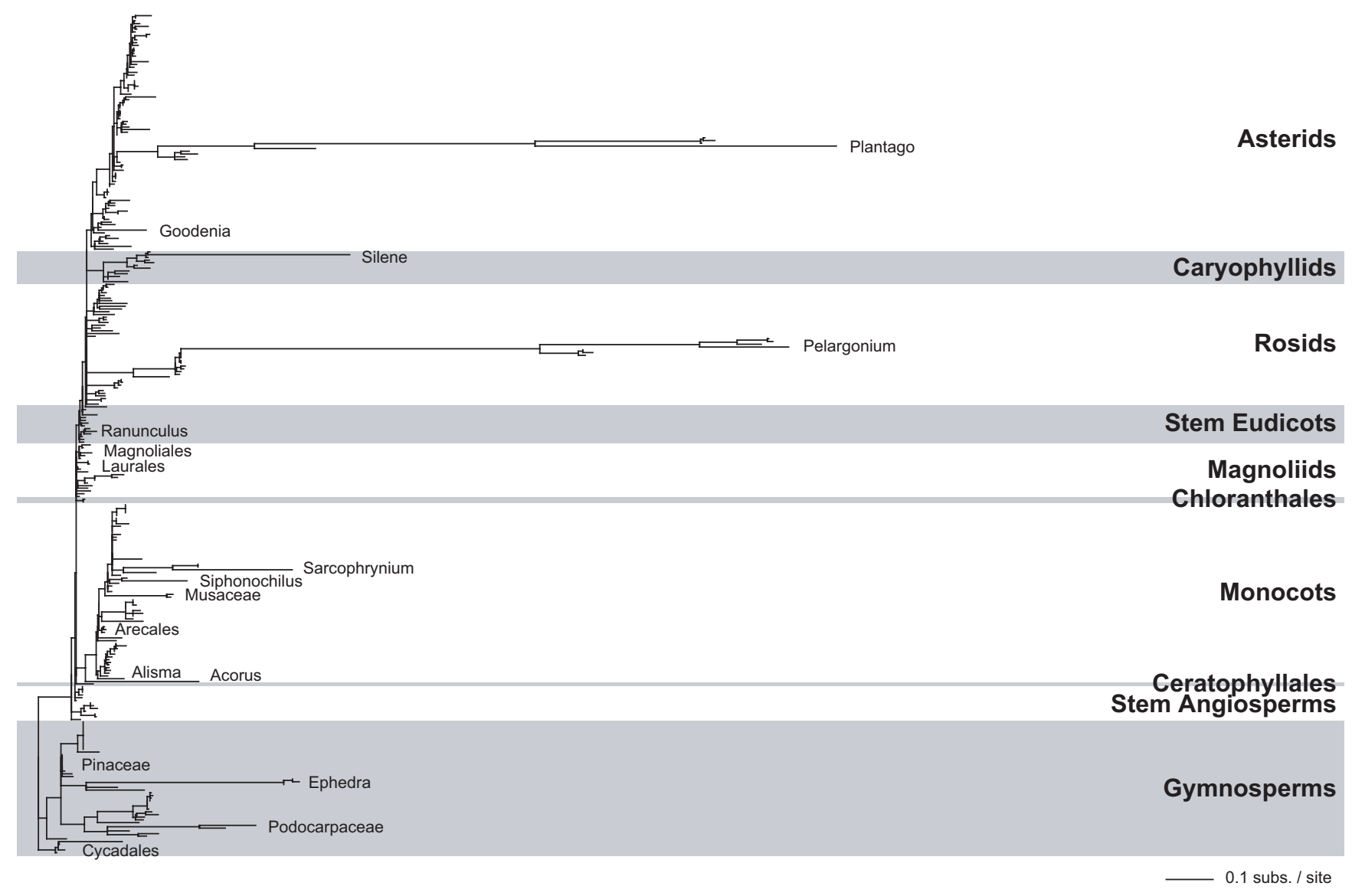

Figure 2

Synonymous sequence divergence for mitochondrial cox $I$. Shown is the $d_{S}$ tree resulting from a likelihood analysis of 306 mitochondrial cox I sequences. Details are as in Figure I.

$\left(d_{\mathrm{N}}\right)$ divergence values were estimated for a subset of taxa from a combined analysis of five mitochondrial protein genes (Fig. 4). Patterns of divergence in the $d_{S}$ tree from the combined analysis (Fig. 4) are similar to those observed for the trees based on analyses of individual protein-coding genes and rDNA (Fig. 5). In all cases, branch lengths are substantially longer for Silene noctiflora, Acorus, Ephedra, and Podocarpus than for most seed plants, but not as long as for Plantago rugelii or Pelargonium hortorum (Figs. 4,5$). d_{\mathrm{N}}$ values are also high for these six species, but they are less pronounced than the increases observed for $d_{\mathrm{S}}$ (Fig. 4 ; note the 10 -fold difference in $d_{\mathrm{S}}$ and $d_{\mathrm{N}}$ scales).

\section{Absolute substitution rates in mitochondrial genes of seed plants}

The length of a branch in a phylogenetic tree, which represents an estimate of the amount of sequence divergence for that lineage, equals the product of the absolute substitution rate(s) and time. Because of this confluence, the rate and time components must be separated in order to make direct comparisons of substitution rates among taxa. We previously described a method to calculate absolute rates of synonymous $\left(R_{\mathrm{S}}\right)$ and nonsynonymous $\left(R_{\mathrm{N}}\right)$ substitution along each branch in a phylogenetic context $[6,7]$, and we employ essentially the same strategy here. However, in this case $d_{S}$ and $d_{N}$ values were estimated from a combined analysis of five mitochondrial protein genes (Fig. 4).

There is a wide range of variation in $R_{\mathrm{S}}$ across seed plants (Table 1). At one extreme, the synonymous rate in Cycas is only 0.015 substitutions per site per billion years (SSB). Slow rates are also seen for several other gymnosperms, including Pinus, Ginkgo, and Zamia. The Cycas value is more than 5-fold lower than the lowest angiosperm rates, from Platanus, Laurus, Liriodendron, Phoenix, and Sambucus. At the other extreme, $R_{\mathrm{S}}$ is equal to 90 SSB for Silene noctiflora. The high rate of synonymous substitution for $S$. noctiflora was recently discovered by a second group as well (DB Sloan, CM Barr, MS Olson, SR Keller, and DR Taylor, personal communication). $R_{\mathrm{S}}$ for $S$. noctiflora is even faster than for those species of Plantago and Pelargo- 


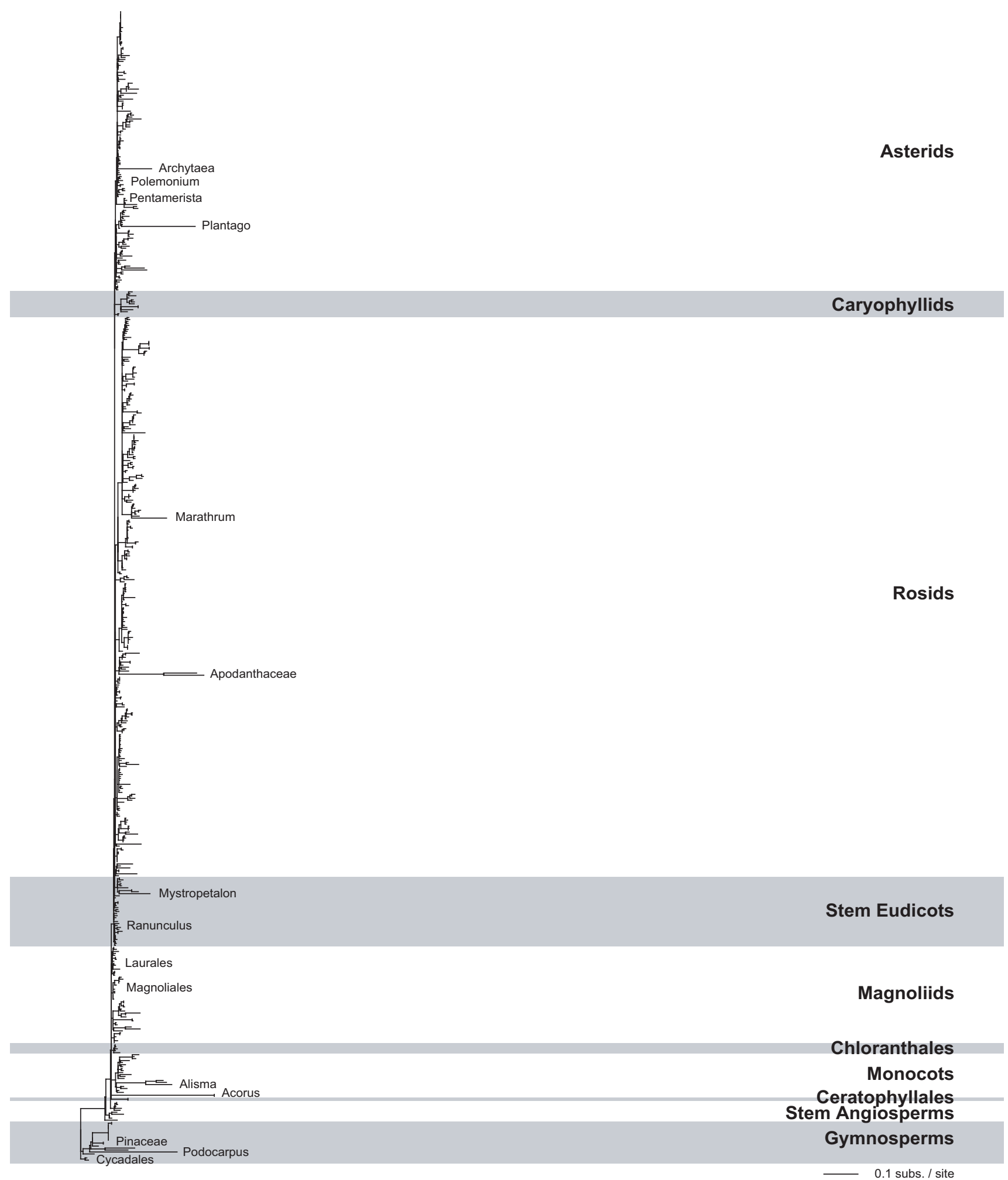

\section{Figure 3}

Synonymous sequence divergence for mitochondrial matR. Shown is the $d_{S}$ tree resulting from a likelihood analysis of 578 mitochondrial matR sequences. Details are as in Figure I. Note that the matR gene has not been isolated from Pelargonium or the fastest members of Plantago. 


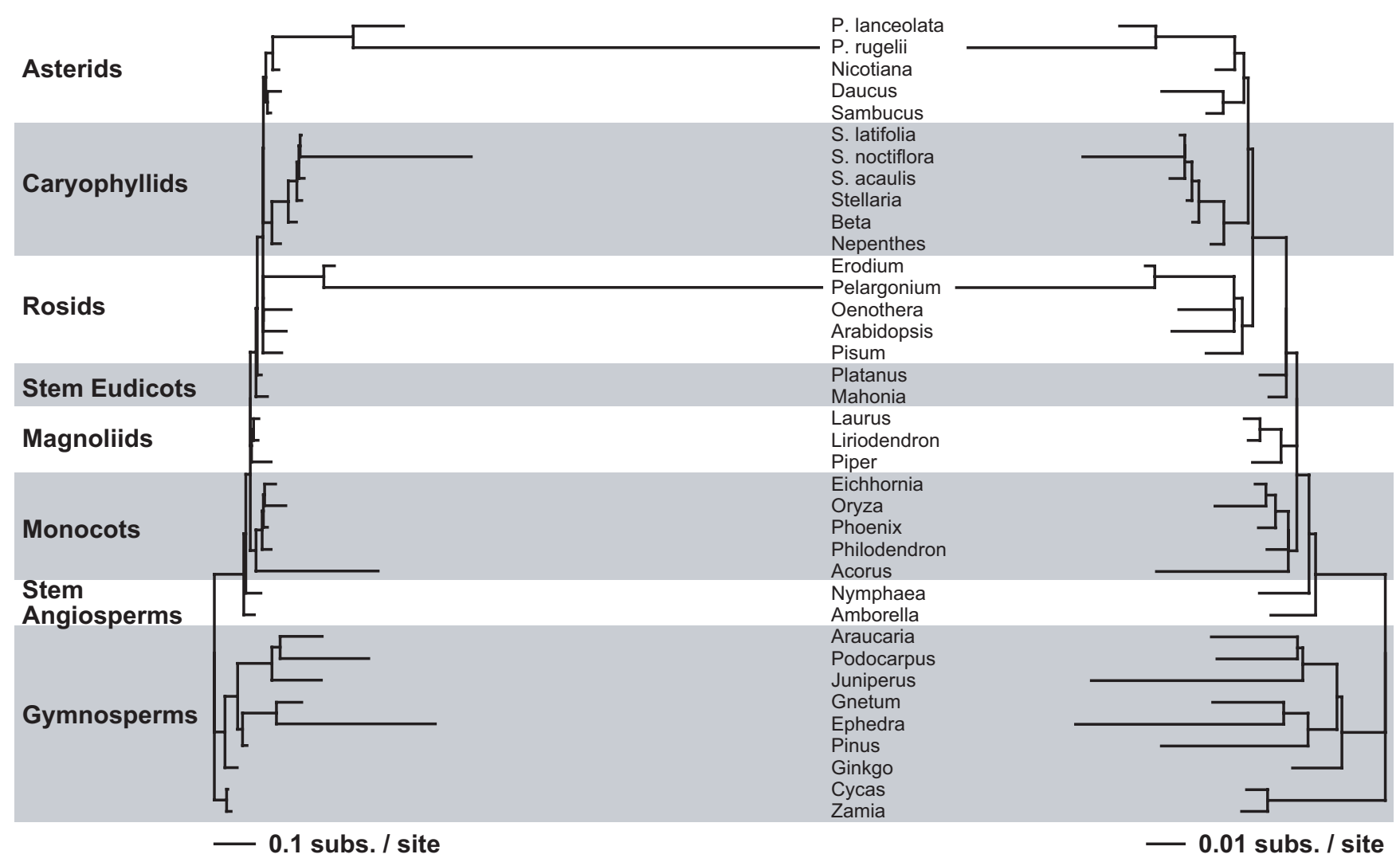

Figure 4

Multigene analysis of synonymous and nonsynonymous sequence divergence. Shown are the $d_{S}\left(\right.$ left) and $d_{N}$ (right) trees resulting from a codon-based likelihood analysis of a combined data set of five mitochondrial genes (atpl, cob, cox $I$, cox2, and cox3). Branch lengths are in units of synonymous substitutions per synonymous site for the $d_{S}$ tree and nonsynonymous substitutions per nonsynonymous site for the $d_{N}$ tree; note the 10 -fold difference in scaling between the two trees. Topological constraints were enforced during the analysis (see methods). Sequences for two to five of the genes analyzed were available for any one plant [see Supp. Table 5 in Additional file 2]. S. = Silene; P. = Plantago.

nium sampled here, but lower than the fastest previously reported angiosperm rate of 166-244 SSB for Plantago media [6]. Between Cycas and P. media, synonymous substitutions vary by a factor of 11,000 to 16,000 across seed plants.

$R_{\mathrm{N}}$ is also variable across seed plants, ranging by a factor of 250 between Liriodendron and S. noctiflora (Table 1). There is a general correlation between $R_{\mathrm{N}}$ and $R_{\mathrm{S}}\left(\mathrm{R}^{2}=\right.$ $0.80)$; however, $R_{\mathrm{N}}$ values are muted relative to their $R_{\mathrm{S}}$ counterparts (Figs. 4 and 6 ). Consequently, $R_{\mathrm{N}} / R_{\mathrm{S}}$ ratios (Table 1 ) are generally lower for species with high $R_{\mathrm{S}}$ (e.g., P. rugelii, $S$. noctiflora, and Pelargonium) and higher for species with low $R_{\mathrm{S}}$ (e.g., Cycas, Pinus, and Platanus). These ratios suggest very different evolutionary environments at the extremes. For $P$. rugelii and Pelargonium, substitutions at synonymous sites occur 25 times more frequently than at nonsynonymous sites, whereas for Pinus and Cycas, nonsynonymous substitutions are actually estimated to occur 2-4 times more often than synonymous substitutions.

It must be noted here that sites of RNA editing were not excluded in the analyses of this study. In plants, RNA editing usually occurs at multiple positions for any mitochondrial transcript $[17,18]$. The retention of edited sites in rate analyses can sometimes bias estimates of nonsynonymous substitution rates, although their presence seems to have little to no effect on synonymous rate estimates $[19,20]$. Indeed, the exclusion of edited sites in gymnosperm $\operatorname{cox} 1$ sequences resulted in a 30 -fold reduction in the nonsynonymous rate [19]. Thus, the high $R_{\mathrm{N}} / R_{\mathrm{S}}$ ratios observed for Pinus and Cycas are probably an artifact of the large number of edited sites present in their genes.

To further explore the dynamics of the rate acceleration within Silene, $R_{\mathrm{N}}$ and $R_{\mathrm{S}}$ for all internal and terminal branches in the Caryophyllales were plotted phylogeneti- 

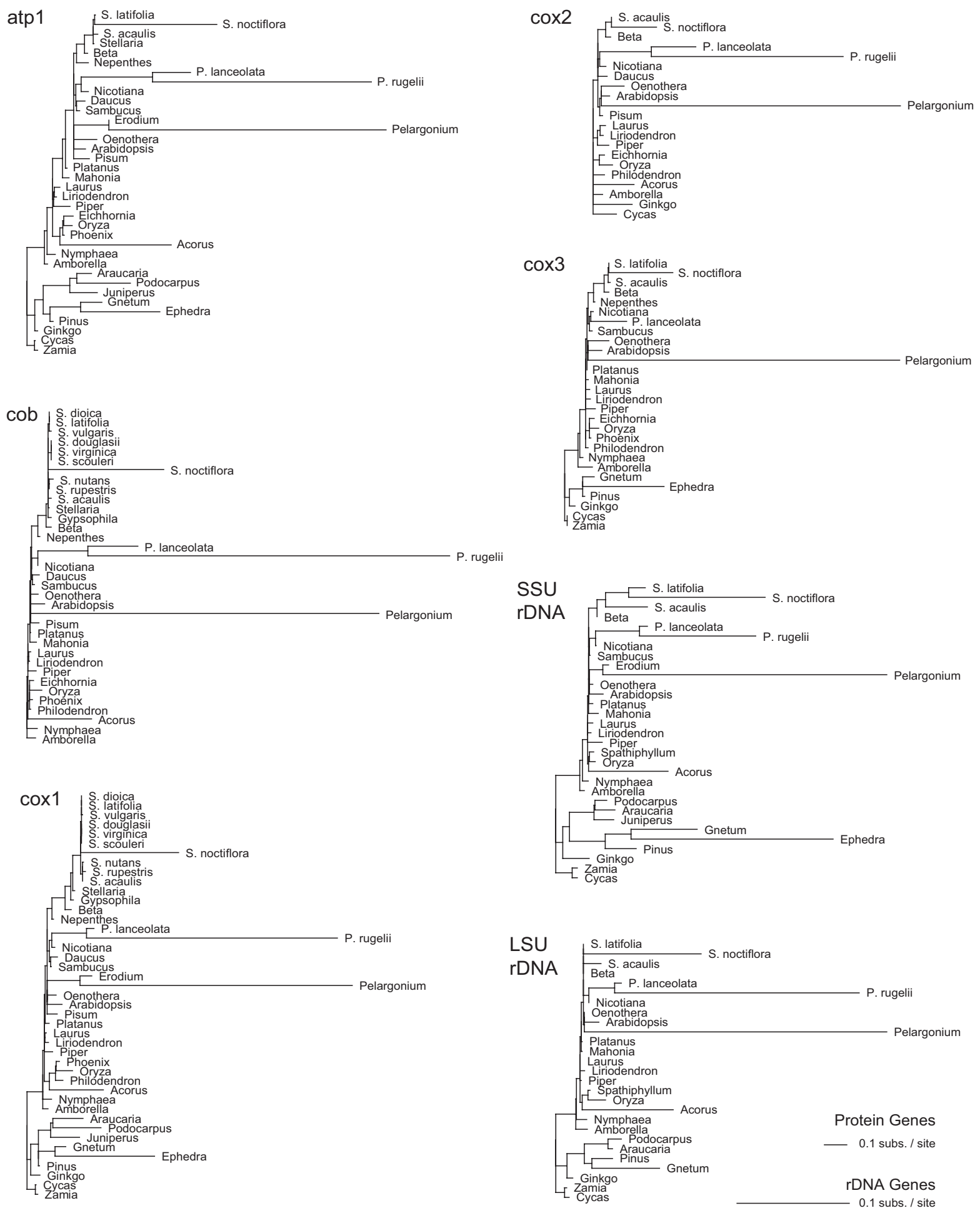

\section{Figure 5}

Individual gene analyses of synonymous sequence divergence. Shown are the $d_{S}$ trees resulting from individual, codonbased likelihood analyses of five mitochondrial protein-coding genes (atpl, cob, cox I, cox2, and $\operatorname{cox} 3$ ) and overall divergence from individual, nucleotide-based likelihood analyses of SSU and LSU rDNA. Branch lengths are in units of synonymous substitutions per synonymous site for the protein-coding genes and in units of substitutions per site for rDNA genes. Topological constraints were enforced during the analysis (see methods). S. = Silene; P. = Plantago. 
Table I: Divergence times, absolute substitution rates, and synonymous to nonsynonymous rate ratios for all terminal branches

\begin{tabular}{|c|c|c|c|c|}
\hline Branch & Time (MYA) & $R_{\mathrm{S}}(\mathrm{SSB})$ & $R_{\mathrm{N}}(\mathrm{SSB})$ & $R_{\mathrm{N}} / \boldsymbol{R}_{\mathrm{S}}$ \\
\hline P. lanceolata & $17 \pm 1$ & $7.71 \pm 2.08$ & $0.53 \pm 0.13$ & 0.07 \\
\hline P. rugelii & $17 \pm 1$ & $69.62 \pm 6.33$ & $2.76 \pm 0.30$ & 0.04 \\
\hline Nicotiana & $82 \pm 4$ & $0.19 \pm 0.06$ & $0.06 \pm 0.02$ & 0.30 \\
\hline Daucus & $89 \pm 5$ & $0.38 \pm 0.06$ & $0.17 \pm 0.03$ & 0.45 \\
\hline Sambucus & $89 \pm 5$ & $0.10 \pm 0.04$ & $0.05 \pm 0.01$ & 0.50 \\
\hline S. latifolia & $5 \pm 1$ & $0.54 \pm 0.38$ & $0.22 \pm 0.12$ & 0.40 \\
\hline S. noctiflora & $5 \pm 1$ & $90.12 \pm 13.94$ & $5.37 \pm 1.00$ & 0.06 \\
\hline S. acaulis & $8 \pm 1$ & $1.55 \pm 0.52$ & $0.45 \pm 0.15$ & 0.29 \\
\hline Stellaria & $19 \pm 4$ & $0.59 \pm 0.24$ & $0.07 \pm 0.06$ & 0.11 \\
\hline Beta & $38 \pm 2$ & $0.56 \pm 0.12$ & $0.04 \pm 0.02$ & 0.07 \\
\hline Nepenthes & $76 \pm 4$ & $0.29 \pm 0.07$ & $0.05 \pm 0.02$ & 0.16 \\
\hline Erodium & $41 \pm 3$ & $0.79 \pm 0.72$ & $0.06 \pm 0.03$ & 0.08 \\
\hline Pelargonium & $41 \pm 3$ & $30.70 \pm 3.07$ & $1.22 \pm 0.17$ & 0.04 \\
\hline Arabidopsis & $100 \pm 4$ & $0.56 \pm 0.07$ & $0.16 \pm 0.02$ & 0.28 \\
\hline Oenothera & $100 \pm 4$ & $0.70 \pm 0.08$ & $0.14 \pm 0.03$ & 0.20 \\
\hline Pisum & $100 \pm 4$ & $0.45 \pm 0.07$ & $0.09 \pm 0.02$ & 0.20 \\
\hline Platanus & $130 \pm 6$ & $0.08 \pm 0.03$ & $0.05 \pm 0.01$ & 0.57 \\
\hline Mahonia & $|3| \pm 6$ & $0.23 \pm 0.05$ & $0.03 \pm 0.01$ & 0.14 \\
\hline Laurus & $133 \pm 6$ & $0.11 \pm 0.03$ & $0.03 \pm 0.01$ & 0.30 \\
\hline Liriodendron & $133 \pm 6$ & $0.09 \pm 0.03$ & $0.02 \pm 0.01$ & 0.24 \\
\hline Piper & $137 \pm 6$ & $0.36 \pm 0.05$ & $0.05 \pm 0.01$ & 0.14 \\
\hline Eichhornia & $86 \pm 4$ & $0.33 \pm 0.08$ & $0.03 \pm 0.01$ & 0.09 \\
\hline Oryza & $86 \pm 4$ & $0.61 \pm 0.08$ & $0.15 \pm 0.03$ & 0.24 \\
\hline Phoenix & $92 \pm 5$ & $0.11 \pm 0.04$ & $0.05 \pm 0.02$ & 0.43 \\
\hline Philodendron & $118 \pm 6$ & $0.19 \pm 0.05$ & $0.04 \pm 0.01$ & 0.23 \\
\hline Acorus & $127 \pm 6$ & $2.45 \pm 0.21$ & $0.26 \pm 0.03$ & 0.11 \\
\hline Nymphaea & $153 \pm 8$ & $0.26 \pm 0.05$ & $0.08 \pm 0.02$ & 0.31 \\
\hline Amborella & $158 \pm 8$ & $0.18 \pm 0.04$ & $0.07 \pm 0.01$ & 0.42 \\
\hline Araucaria & $245 \pm 0$ & $0.43 \pm 0.07$ & $0.09 \pm 0.01$ & 0.20 \\
\hline Podocarpus & $245 \pm 0$ & $0.93 \pm 0.10$ & $0.08 \pm 0.02$ & 0.09 \\
\hline Juniperus & $267 \pm 8$ & $0.47 \pm 0.06$ & $0.20 \pm 0.02$ & 0.43 \\
\hline Pinus & $286 \pm 13$ & $0.04 \pm 0.02$ & $0.13 \pm 0.02$ & 3.27 \\
\hline Gnetum & $169 \pm 27$ & $0.38 \pm 0.10$ & $0.11 \pm 0.03$ & 0.28 \\
\hline Ephedra & $169 \pm 27$ & $2.36 \pm 0.43$ & $0.31 \pm 0.06$ & 0.13 \\
\hline Ginkgo & $315 \pm 5$ & $0.10 \pm 0.02$ & $0.04 \pm 0.01$ & 0.39 \\
\hline Cycas & $179 \pm 9$ & $0.02 \pm 0.01$ & $0.03 \pm 0.01$ & 1.94 \\
\hline Zamia & $179 \pm 9$ & $0.06 \pm 0.02$ & $0.03 \pm 0.01$ & 0.59 \\
\hline
\end{tabular}

cally (Fig. 7). Only the branch leading to S. noctiflora shows a substantial increase in rate, indicating that the rate acceleration occurred within the last five million years, subsequent to the divergence of $S$. noctiflora from the other two species of Silene included in this analysis. To determine whether any other species of Silene show elevated substitution levels, seven additional species were sampled for the genes $c o b$ and cox 1 . However, none of the additional taxa appear to be any more closely related to $S$. noctiflora than is S. latifolia [see Supp. Fig. 1 in Additional file 1], and none of their sequences are unusually divergent (Fig. 5). Because these additional species did not provide further information on the timing of the rate acceleration, they were not included in the absolute rates analyses in Figure 7.

The frequency of mitochondrial RNA editing was shown to be highly reduced in Pelargonium and other Gera- niaceae, and this observation was postulated to be potentially related to the increase in their mitochondrial synonymous substitution rate [7]. To discover whether the frequency of editing is also reduced in $S$. noctiflora, edit sites were predicted using the online resource PREP-Mt [21] for the six taxa shown in Figure 7 (Table 2). Contrary to the situation in the Geraniaceae, there appears to be little correlation between substitution rate and editing frequency in Silene. The number of predicted edit sites in $S$. noctiflora is quite similar to the other two species of Silene and to Stellaria, despite the roughly 100-fold increase in the synonymous substitution rate specific to $S$. noctiflora mitochondrial genes.

\section{Discussion Absolute substitution rates}

Previous studies on synonymous substitution rates found that plant mitochondrial sequences evolve very slowly [1- 


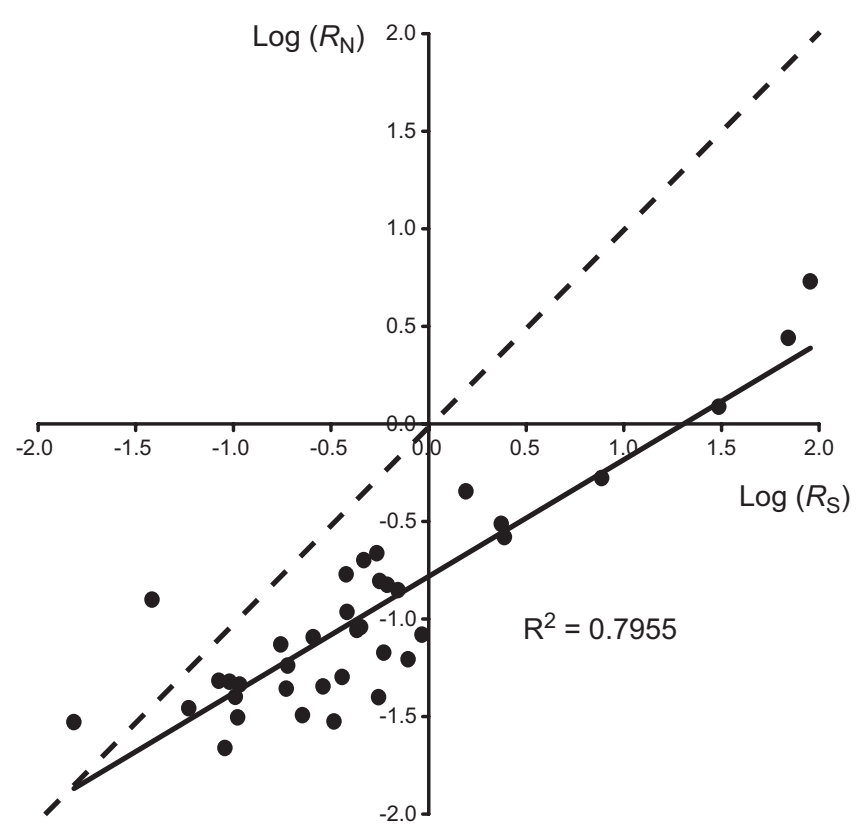

Figure 6

Correlation between absolute synonymous and nonsynonymous rates. Absolute rates of nonsynonymous $\left(R_{N}\right)$ and synonymous $\left(R_{S}\right)$ substitution from Table 4 were logtransformed and then plotted for each terminal branch. $\log \left(R_{\mathrm{S}}\right)$ values are on the horizontal axis and $\log \left(R_{\mathrm{N}}\right)$ values are on the vertical axis. The solid black line is a linear regression of the data. The broken line signifies an $R_{\mathrm{N}}$ to $R_{\mathrm{S}}$ ratio of I.

5]. For the most part, the results presented in Figures 1, 2, 3 confirm these earlier findings. Roughly $80-90 \%$ of the sampled species have normally low levels of sequence divergence at synonymous sites. However, a surprising number of species depart from the "normal" mitochondrial pattern. At one end of the spectrum, there are several angiosperm (e.g., Arecales, Chloranthales, and Laurales) and gymnosperm (e.g., Cycadales and Pinaceae) groups whose genes are unusually conserved at synonymous sites. At the opposite end are taxa such as Plantago, Pelargonium, and $S$. noctiflora with long branches for multiple genes. Many additional species have long branches for a single gene, but independent sequences are needed to confirm that these long branches are replicable and not artifactual (also see next section). On the whole, these results suggest that while most lineages of plant mitochondria do indeed possess generally low synonymous substitution rates, it's increasingly naive and misleading to categorize all plants in this way, and that synonymous substitution rates are, for reasons still unclear, exceptionally fluid and variable in plant mitochondria.

Because the analyses in Figures 1, 2, 3 used almost all species available in GenBank (some species were lost during data filtration; see methods), they provide a generally unbiased representation of rate variation across seed plants. However, for comparisons among species, branch lengths alone are inadequate because they are a function of absolute substitution rate(s) and time. In other words, equally long branches do not necessarily equate to equally fast absolute substitution rates. For example, $d_{\mathrm{S}}$ for $S$. noctiflora, Acorus, and Ephedra are quite similar, but $R_{\mathrm{S}}$ for $S$. noctiflora is almost 40 times higher than for Acorus and Ephedra (Table 1). Indeed, the synonymous rate for $S$. noctiflora is even faster than for the species of Plantago and Pelargonium examined here (Table 1), despite the much shorter branch length for S. noctiflora (Fig. 4).

These findings highlight the importance of taxon sampling in these analyses. The increased sampling of Silene species allowed us to pinpoint the rate acceleration in $S$. noctiflora to a 5 million year period. Similarly, sampling of multiple taxa within Plantago in a previous study on synonymous rates identified one species, $P$. media, with $R_{\mathrm{S}}$ a few times higher than for $S$. noctiflora over a similar time span [6]. The finding of extensive variation in synonymous substitution rates within these two genera suggests that there is likely to be much more rate variation in plant mitochondrial genomes than is already apparent. Increasingly dense sampling of species is likely to uncover additional examples of rate variation. Calculation of absolute substitution rates may identify further examples, because some increases may be so recent that sequence divergence is still rather low and not readily apparent in phylogenetic trees.

Unfortunately, for Acorus and Ephedra there is little chance that increased sampling will provide a better estimate of the timing and magnitude of the rate acceleration, despite the fact that their increased divergence levels are spread over more than 100 million years. For Acorus, the genus encompasses only three or four species and they all appear to be very similar at the molecular level (see three species in Figure 1 and two species in Figure 3). Furthermore, there are no other genera in the Acorales to break up the time period from the base of monocots to the diversification of Acorus [22]. With over 60 species in Ephedra, it may be possible to more finely dissect the dynamics of rate evolution in this genus. However, like the situation in Acorus, the species included here show very little molecular divergence (see three species in Figure 2) and there are no other genera to break up the long time interval between the crown and stem group nodes of Ephedra [22].

Among-species comparisons reveal that synonymous substitution rates vary by four orders of magnitude across seed plants, from a low of 0.015 SSB in Cycas (Table 1) to a high of 166-244 SSB in Plantago media [6]. Excluding RNA viruses [23], this is, to our knowledge, by far the larg- 


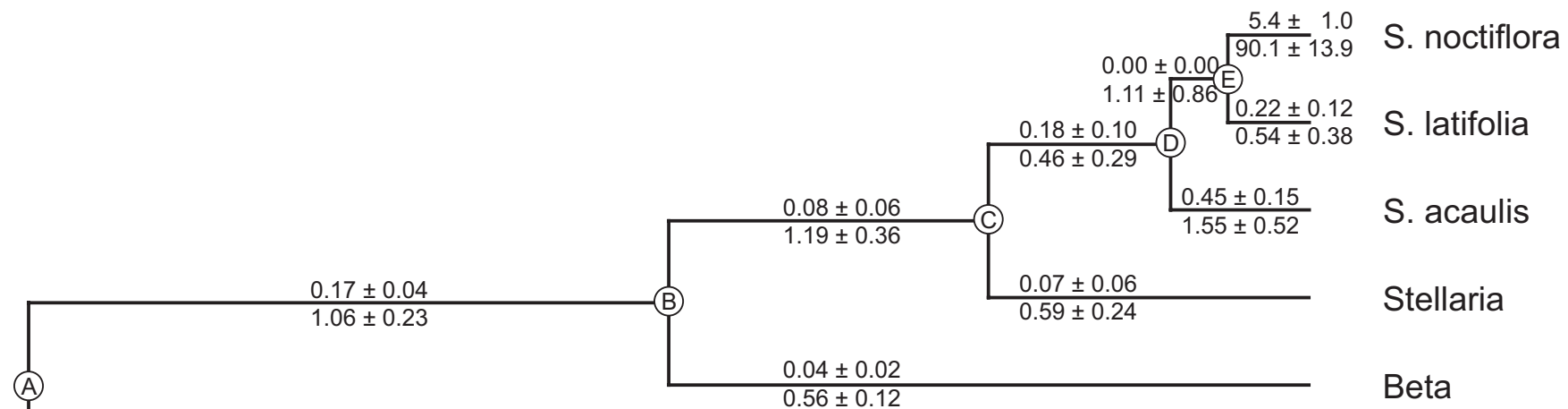

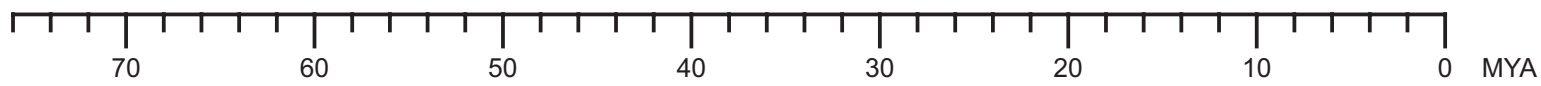

Figure 7

Absolute substitution rates in Caryophyllales. Absolute rates of nonsynonymous $\left(R_{\mathrm{N}}\right)$ and synonymous $\left(R_{\mathrm{S}}\right)$ substitution are plotted above and below, respectively, each branch of the chronogram. $R_{\mathrm{N}}$ and $R_{\mathrm{S}}$ values were calculated as in Table I, using branch lengths from Figure 4 and divergence times for nodes $A$ and $B$ from Wikstrom et al [45] and for nodes $C$, $D$, and $E$ from Supplementary Figure I [see Additional file I]. MYA = million years ago.

est range of synonymous rates known for any phylogenetic group and genome, as well as both the lowest and highest estimated rates. To put this range in perspective, the amount of mitochondrial sequence divergence found in Cycas after almost 180 million years of evolution would have taken $P$. media on average only about 15 thousand years to accumulate. These results highlight the advantage of estimating rates in a phylogenetic context. Traditional pairwise methods average rates between two lineages, thereby masking any lineage-specific rate differences. A pairwise comparison between $S$. noctiflora at 90 SSB and $S$. latifolia at $0.54 \mathrm{SSB}$ would result in an averaging of their very different rates to $\sim 45 \mathrm{SSB}$, which doesn't reflect either of their actual rates very well. Similarly, the low rate found in Cycas (0.015 SSB) wouldn't be quite as low in a pairwise comparison with Zamia (0.059 SSB) leading to a pairwise rate of $0.037 \mathrm{SSB}$. The phylogenetic estimates of substitution rates indicate a 6000 -fold range of variation

Table 2: Number of RNA editing sites predicted by PREP-Mt (C $=0.5)$

\begin{tabular}{lccccc}
\hline Species & atpl & cob & coxl & cox2 & cox3 \\
\hline S. noctiflora & 0 & 5 & 1 & 2 & 1 \\
S. acaulis & 2 & 6 & 0 & 2 & 1 \\
S. latifolia & 2 & 7 & 0 & - & 1 \\
Stellaria & 1 & 7 & 1 & - & - \\
Beta & 3 & 9 & 0 & 8 & 2 \\
Nepenthes & 4 & 12 & 1 & - & 4 \\
\hline
\end{tabular}

between Cycas and S. noctiflora versus a 1000-fold range based on the pairwise estimates.

Although substitution rates are high for mitochondrial genes of S. noctiflora and Acorus, chloroplast and nuclear genes from these species do not show any increase in sequence divergence [see Supp. Fig. 1 in Additional file 1] [24-27]. Thus, the rate acceleration in these species is confined to the mitochondrial genome, as seen also in Plantago and Pelargonium [6,7]. In those studies, defects in mitochondrial DNA replication or repair were speculated to be, among a wide range of processes potentially affecting the mitochondrial mutation rate, the most likely causes of such severe, mitochondrial-specific increases in substitution rates. Similar factors may also be at work in S. noctiflora and Acorus. In contrast, in Ephedra, chloroplast and nuclear sequences are also divergent $[27,28]$. This points to a factor acting at the organismal level, such as generation time, that would affect rates in all three genomes, although mitochondrial-specific forces may be at work as well. Some studies [16,29-31] have detected moderate, correlated differences in synonymous substitution rates across all three plant genomes and have attributed these to generation time effects $[16,29,30]$, paternal transmission of organelles [31], or correlated substitution and speciation rates $[32,33]$.

\section{Within-plant variation in sequence divergence}

One surprising finding from the broad-scale analyses is the number of plant lineages with levels of synonymous 
site divergence that appear to be relatively high for some genes and low for others (Figs. 1, 2, 3). In most cases, the basis for this apparent within-plant rate heterogeneity is unknown, and as emphasized below, some of these cases are likely to be or potentially are the result of error of various kinds. Others, however, have a possible biological underpinning. One likely biological explanation is horizontal gene transfer, which occurs surprisingly frequently between mitochondrial genomes of unrelated plants $[34,35]$. Transfers between genomes with highly different substitution rates and accumulated sequence divergence could easily account for some of the within-plant, geneto-gene differences shown in Figs. 1, 2, 3 and indeed is suspected to account for one particular case. Two members of the Apodanthaceae, Pilostyles and Apodanthes, have high levels of divergence for matR. The Apodanthes atp1 gene is similarly divergent, but atp 1 from Pilostyles is not. Phylogenetic analysis of the atp1 gene from Pilostyles suggests the possibility that this gene may have been acquired via horizontal transfer from a plant with normally low mitochondrial rates [12].

Another potential biological explanation for differences in synonymous rates between genes from the same plant is that they are located in different genetic compartments that possess different substitution rates. With the exception of Plantago and Pelargonium [6,7] and probably some of the new cases of high-rate mitochondrial genomes reported in this study, synonymous rates are much lower in mitochondrial than nuclear genomes in plants [1-4]. Functional transfer of a mitochondrial gene to the nucleus will thus usually lead to much higher rates of sequence divergence. This possibility is exciting because there has been no report of functional nuclear transfer for atp1, matR, or $\operatorname{cox} 1$ in any plant. This includes no evidence for loss of these three genes from the mitochondrial genomes of 280 diverse angiosperms examined by Southern blot hybridization, whereas 16 other genes were inferred to be frequently lost from the mitochondrial genome and, equally frequently, functionally transferred to the nucleus [36].

A third intriguing possibility is that synonymous substitution rates vary across regions of the mitochondrial genome. In the chloroplast genome of angiosperms, synonymous rates are known to be a few-fold higher in single-copy regions than in a large inverted-repeat region $[1,37]$, and of perhaps greater relevance, a small region with higher accelerated synonymous rates has recently been discovered in the chloroplast of one lineage of legumes (KH Wolfe, personal communication). Gene-togene variation in the mitochondrial mutation rate was also recently observed in populations of Silene vulgaris $[38,39]$.
There are also several non-biological reasons why divergence levels for a particular species vary between genes. Sequencing errors will artificially inflate sequence divergence. The Pennisetum atp1 sequence is a likely example. Including the atp 1 sequence, eight mitochondrial genes were generated for Pennisetum from the same unpublished study (GenBank: AF511559-AF511569), and all show similar characteristics including frameshifting indels and a large number of nucleotide substitutions (data not shown). Most likely, these unpublished sequences are full of errors, and this is the cause of the apparently anomalous divergence of atp1 in Pennisetum.

Misidentification of the correct phylogenetic position of a sequence/organism could also lead to artifactually long branches (recall that topological constraints were enforced for $d_{\mathrm{S}}$ branch length estimation). For example, two cases were found where the taxonomic sources of sequences were swapped. In the first case, a cox 1 sequence whose source is listed as Austrobaileya (GenBank: AF193954) is more similar to an independent Ceratophyllum sequence than to other Austrobaileyales sequences, while a sequence annotated as Ceratophyllum (GenBank: AF193945) is actually more Austrobaileya-like. These two GenBank files have now been corrected. A similar mix-up occurred between a Ginkgo-like sequence annotated as Cabomba (GenBank: X94585) and a Cabomba-like sequence identified as Ginkgo (GenBank: X94587). In each example, the mislabeled sequences were generated from the same study and probably reflect a mix-up that occurred during the database submission process. They were easily identifiable (and were excluded from the final analyses) because they appeared as uncharacteristically long branches relative to independent sequences of the same gene from the same genus.

The use of topological constraints in the analyses may similarly lead to spuriously long branches. To evaluate this possibility, unconstrained analyses were performed on the four data sets used for Figures 1, 2, 3, 4 [see Supp. Figs. 2-3 in Additional file 1]. As can be seen, almost all long branches in the constrained analyses remain long in the unconstrained analyses. However, the use of topological constraints apparently led to an erroneously long branch for Archytaea matR and for Pennisetum atp1. Archytaea was constrained as an asterid in the Ericales (based on GenBank taxonomy), but it is actually a rosid in the Malpighiales [22]. In the unconstrained analysis for matR, Archytaea groups properly within the Malpighiales and exhibits a more normal branch length [see Supp. Fig. 2 in Additional file 1]. The long branch for Pennisetum atp1 also greatly diminishes in the unconstrained analyses [see Supp. Fig. 2 in Additional file 1]. As mentioned above, the long Pennisetum branch may result from a poor-quality sequence read. Alternatively, the sequence may have been 
acquired via horizontal gene transfer, or the DNA sample was derived from an organism mistakenly identified as Pennisetum.

These examples underscore the need for independent sequencing to verify that any and all cases of putative intragenomic variation in synonymous substitution rates are real and not the result of human error. For those cases that are validated, it will be interesting to see whether any turn out to reflect noteworthy events in mitochondrial evolution, such as gene- or region-specific differences in substitution rates within a mitochondrial genome, horizontal gene transfer, or the functional transfer to the nucleus of a gene that has never known to have been sotransferred in plants (atp1 or matR) or even among all eukaryotes ( $\operatorname{cox} 1)$.

\section{Conclusion}

In this study, we have measured synonymous substitution rates in a phylogenetic context and uncovered numerous independent examples of rate increase and decrease. These results demonstrate that the synonymous substitution rate in plant mitochondria is a more variable character than previously appreciated. The extremes of synonymous substitution rates measured here constitute by far the largest known range of rate variation for any group of organisms, yet there is no obvious explanation for these divergent patterns. Future studies are required to understand the evolutionary processes driving these patterns.

\section{Methods \\ Molecular techniques}

Total genomic DNA was extracted and purified from fresh leaves using a CTAB protocol [40] or using the DNeasy Plant Mini Kit (QIAGEN) according to manufacturer's instructions. Genes were amplified by polymerase chain reaction using a PTC-200 thermocycler (MJ Research) and gene-specific primers [see Supp. Table 1 in Additional file 2]. Each reaction was performed using 35 cycles of $30 \mathrm{sec}$ at $94^{\circ} \mathrm{C}, 30 \mathrm{sec}$ at $50^{\circ} \mathrm{C}$, and $2.0-2.5 \mathrm{~min}$ at $72^{\circ} \mathrm{C}$, with an initial step of $3 \mathrm{~min}$ at $94^{\circ} \mathrm{C}$ and a final step of $10 \mathrm{~min}$ at $72^{\circ} \mathrm{C}$. Polymerase chain reaction products were purified using ExoSAP-IT (United States Biochemical) and then sequenced on both strands using an ABI 3730 (Applied Biosystems) at the Indiana Molecular Biology Institute. Sequences newly determined in this study were deposited in GenBank under accessions EF547202EF547251. Additional nucleotide sequences used in this study were obtained from GenBank [see Supp. Tables 2-7 in Additional file 2].

\section{Survey of mitochondrial sequence divergence}

Similarity searches were performed with blastn against the nonredundant GenBank database using the atp1, cox1, and matR genes from Arabidopsis thaliana as queries (GenBank: Y08501). Limitations were enforced during the searches such that only hits from the Spermatophyta (gymnosperms and angiosperms) with an e-value less than $1 \mathrm{e}^{-10}$ were included. Hits less than $200 \mathrm{bp}$ in length, multiple hits to the same species, pseudogenes, and cDNA sequences were removed from the blast results, while sequences generated in this study were added. Sequences were aligned using ClustalX and manually adjusted when necessary. Poorly alignable regions were excluded from the data sets, as were regions with gaps in the majority of taxa. These data sets (and all others used in the paper) are available [see Additional file 3].

For each surveyed gene, a ML topology was determined with PAUP*. To ensure completion of the ML analysis, several time-saving steps were taken. First, a partially-constrained topology was enforced. The topology was constrained such that all sequences within a family (as defined by GenBank taxonomy) were forced to be monophyletic. Relationships among families were constrained according to information from the Angiosperm Phylogeny Website [22]. Relationships among the major seed plant groups were constrained according to the 9-gene $\mathrm{ML}$ analysis of Qiu et al [41]. Second, ML parameters were fixed to values estimated from a neighbor-joining prior to the ML analysis. Third, the ML analysis used the $\mathrm{HKY}+\mathrm{G}+\mathrm{I}$ model with four rate categories rather than the more parameter-rich GTR+G+I model. The ML topology was identified by a heuristic search starting from the aforementioned neighbor-joining tree and using the TBR branch-swapping algorithm and the MULTREES option.

To determine whether the PAUP* time-saving strategies had an effect on the results of this study, a ML topology was also determined for each gene using RAxML version 2.2.3 [42]. In contrast to the PAUP* analysis, no topological constraints were enforced, the GTR+G model was used (GTR+G+I is not available in RAxML), the search started from a maximum parsimony tree, and the $\mathrm{ML}$ parameters were estimated during the ML analysis. For each gene, 10 replicates were performed starting from 10 different randomized parsimony trees. The initial rearrangement setting was fixed to 10 for all analyses.

Using the ML tree topologies generated above for each gene, $d_{\mathrm{S}}$ branch lengths were estimated in HyPhy version 0.99 for UNIX [43] using the MG94W9 codon model [15] and allowing for independent $d_{\mathrm{N}}$ and $d_{\mathrm{S}}$ values for each branch (the local parameters option). The $d_{\mathrm{S}}$ trees using the PAUP* topologies are shown in Figures 1, 2, 3, and the $d_{\mathrm{S}}$ trees based on RAxML topologies are shown in Supplementary Figure 2 [see Additional file 1]. 


\section{Estimation of absolute substitution rates}

Absolute substitution rates were calculated for a subset of the taxa in the rate survey using methods that have been described previously [6,7]. Briefly, divergence times within Caryophyllaceae [see Supp. Fig. 1B in Additional file 1] were estimated by penalized likelihood with the program r8s [44], using an ML tree from an analysis of the chloroplast gene matK [see Supp. Fig. 1A in Additional file 1] and 38 million years for the split between Caryophyllaceae and Amaranthaceae as a calibration point [45]. Standard errors for Caryophyllaceae divergence times were estimated from 100 bootstrap replicates. Divergence times and associated errors for Plantago, for the remaining angiosperms, and for gymnosperms were taken from the analyses of Cho et al [6], Wikstrom et al [45], and Magallon and Sanderson [27], respectively. The $d_{\mathrm{S}}$ and $d_{\mathrm{N}}$ trees in Figures 4 and 5 were estimated from individual and combined data sets of five mitochondrial protein-coding genes (atp1, cob, cox1, cox2, cox3) using HyPhy as described in the previous section on a topology constrained according to the Angiosperm Phylogeny Website [22]. Standard errors for $d_{\mathrm{S}}$ and $d_{\mathrm{N}}$ were estimated from 100 bootstrap replicates. $R_{\mathrm{S}}$ and $R_{\mathrm{N}}$ values for each branch were calculated by dividing the mitochondrial sequence divergences from the combined data set by the elapsed time along that branch (Table 1).

\section{Authors' contributions}

JPM, PT, and JSG generated sequence data. JPM and JDP designed the seed plant rate survey and interpreted its results. JPM, PT, LFD, and JDP designed the Silene rates study and interpreted its results. JPM ran all analyses and prepared all figures and tables. JPM and JDP wrote the manuscript. All authors read and approved the final manuscript.

\section{Additional material}

\section{Additional file 1}

Supplementary figures. Supplementary Figure 1 shows the phylogenetic and divergence time analysis for Caryophyllales. Supplementary Figure 2 shows unconstrained analyses of the data sets used for Figures 1, 2, 3. Supplementary Figure 3 shows an unconstrained analysis of the data set used for Figure 4. Supplementary Figures 4-6 show the $\mathrm{d}_{S}$ trees in Figures 1, 2, 3 at an expanded scale and with all taxon names included. Click here for file

[http://www.biomedcentral.com/content/supplementary/14712148-7-135-S1.pdf]

\section{Additional file 2}

Supplementary tables. Supplementary Table 1 lists the primers used in this study. Supplementary Tables 2-7 list the taxon names and GenBank accession numbers of all sequence used in this study.

Click here for file

[http://www.biomedcentral.com/content/supplementary/14712148-7-135-S2.pdf]

\section{Additional file 3}

Data sets. Data sets 1-7 are in nexus format and contain sequences used in the analyses of atp1, cox1, matR, 5-Gene combined, SSU rDNA, LSU $r D N A$, and matK, respectively. These seven data sets are provided as a tar archive compressed with gzip.

Click here for file

[http://www.biomedcentral.com/content/supplementary/14712148-7-135-S3.tgz]

\section{Acknowledgements}

The authors thank Chris Parkinson for providing the Alisma cox I sequence and Sergei Kosakovsky Pond for helpful assistance with the HyPhy software. This work was supported by NIH research grant GM-706 I 2 (to JDP), NSF grant DEB-021097I (to LFD), and a Fulbright/Nord Pas de Calais Region Award (to PT).

\section{References}

I. Wolfe KH, Li WH, Sharp PM: Rates of nucleotide substitution vary greatly among plant mitochondrial, chloroplast, and nuclear DNAs. Proc Natl Acad Sci USA 1987, 84:9054-9058.

2. Wolfe $\mathrm{KH}$ : Molecular evolution of plants: more genomes, fewer generalities. In Molecular Genetics of Photosynthesis Edited by: Andersson B, Salter AH, Barber J. Oxford: IRL Press; 1996:45-57.

3. Gaut BS: Molecular clocks and nucleotide substitution rates in higher plants. In Evolutionary Biology Volume 30. Edited by: Hecht MK, Maclntyre RJ, Clegg MT. New York: Plenum Press; 1998:93-120.

4. Muse SV: Examining rates and patterns of nucleotide substitution in plants. Plant Mol Biol 2000, 42:25-43.

5. Palmer JD, Herbon LA: Plant mitochondrial DNA evolves rapidly in structure, but slowly in sequence. J Mol Evol 1988, 28:87-97.

6. Cho Y, Mower JP, Qiu YL, Palmer JD: Mitochondrial substitution rates are extraordinarily elevated and variable in a genus of flowering plants. Proc Natl Acad Sci USA 2004, 10 I: I 774 I- 17746.

7. Parkinson CL, Mower JP, Qiu YL, Shirk AJ, Song K, Young ND, DePamphilis CW, Palmer JD: Multiple major increases and decreases in mitochondrial substitution rates in the plant family Geraniaceae. BMC Evol Biol 2005, 5:73.

8. Bakker FT, Breman F, Merckx V: DNA sequence evolution in fast evolving mitochondrial DNA nad l exons in Geraniaceae and Plantaginaceae. Taxon 2006, 55:887-896.

9. Bowe LM, Coat G, dePamphilis CW: Phylogeny of seed plants based on all three genomic compartments: extant gymnosperms are monophyletic and Gnetales' closest relatives are conifers. Proc Natl Acad Sci USA 2000, 97:4092-4097.

10. Chaw SM, Parkinson CL, Cheng Y, Vincent TM, Palmer JD: Seed plant phylogeny inferred from all three plant genomes: monophyly of extant gymnosperms and origin of Gnetales from conifers. Proc Natl Acad Sci USA 2000, 97:4086-409I.

II. Davis JI, Stevenson DW, Peterson G, Seberg O, Campbell LM, Freudenstein JV, Goldman DH, Hardy CR, Michelangeli FA, Simmons MP, Specht CD, Vergara-Silva F, Gandolfo M: A phylogeny of the monocots, as inferred from rbcL and atpA sequence variation, and a comparison of methods for calculating jackknife and bootstrap values. Syst Bot 2004, 29:467-5I 0 .

12. Nickrent DL, Blarer A, Qiu YL, Vidal-Russell R, Anderson FE: Phylogenetic inference in Rafflesiales: the influence of rate heterogeneity and horizontal gene transfer. BMC Evol Biol 2004, 4:40. 
13. Petersen G, Seberg O, Davis II, Stevenson DW: RNA editing and phylogenetic reconstruction in two monocot mitochondrial genes. Taxon 2006, 55:87I-886

14. Qiu YL, Li L, Hendry T, Li R, Taylor DW, Issa MJ, Ronen AJ, Vekaria $M L$, White AM: Reconstructing the basal angiosperm phylogeny: evaluating information content of the mitochondria genes. Taxon 2006, 55:837-856.

15. Muse SV, Gaut BS: A likelihood approach for comparing synonymous and nonsynonymous nucleotide substitution rates, with application to the chloroplast genome. Mol Biol Evol 1994, I I:7| 5-724.

16. Eyre-Walker A, Gaut BS: Correlated rates of synonymous site evolution across plant genomes. Mol Biol Evol 1997, I 4:455-460.

17. Giege P, Brennicke A: RNA editing in Arabidopsis mitochondria effects 44 I $\mathbf{C}$ to $\mathbf{U}$ changes in ORFs. Proc Natl Acad Sci USA 1999, 96: I5324- I5329.

18. Mower JP, Palmer JD: Patterns of partial RNA editing in mitochondrial genes of Beta vulgaris. Mol Genet Genomics 2006 276:285-293.

19. Lu MZ, Szmidt AE, Wang XR: RNA editing in gymnosperms and its impact on the evolution of the mitochondrial coxl gene. Plant Mol Biol 1998, 37:225-234.

20. Lopez L, Picardi E, Quagliariello C: RNA editing has been lost in the mitochondrial cox3 and rps/3 mRNAs in Asparagales. Biochimie 2007, 89:159-167.

21. Mower JP: PREP-Mt: predictive RNA editor for plant mitochondrial genes. BMC Bioinformatics 2005, 6:96.

22. Angiosperm Phylogeny Website [http://www.mobot.org/ MOBOT/research/APweb/]

23. Hanada K, Suzuki Y, Gojobori T: A large variation in the rates of synonymous substitution for RNA viruses and its relationship to a diversity of viral infection and transmission modes. Mol Biol Evol 2004, 2 I: 1074-1080.

24. Oxelman B, Liden M: Generic Boundaries in the tribe Sileneae (Caryophyllaceae) as inferred from nuclear rDNA sequences. Taxon 1995, 44:525-542.

25. Mathews S, Donoghue MJ: The root of angiosperm phylogeny inferred from duplicate phytochrome genes. Science 1999, 286:947-950.

26. Popp M, Oxelman B: Evolution of a RNA polymerase gene family in Silene (Caryophyllaceae) - incomplete concerted evolution and topological congruence among paralogues. Syst Biol 2004, 53:9|4-932.

27. Magallon SA, Sanderson MJ: Angiosperm divergence times: the effect of genes, codon positions, and time constraints. Evolution 2005, 59: I653-1670.

28. Hajibabaei M, Xia J, Drouin G: Seed plant phylogeny: Gnetophytes are derived conifers and a sister group to Pinaceae. Mol Phylogen Evol 2006, 40:208-217.

29. Laroche J, Li P, Maggia L, Bousquet J: Molecular evolution of angiosperm mitochondrial introns and exons. Proc Natl Acad Sci USA 1997, 94:5722-5727.

30. Laroche J, Bousquet J: Evolution of the mitochondrial rps3 intron in perennial and annual angiosperms and homology to nad5 intron I. Mol Biol Evol 1999, 1 6:44 I-452.

31. Whittle CA, Johnston MO: Male-driven evolution of mitochondrial and chloroplastidial DNA sequences in plants. Mol Biol Evol 2002, I 9:938-949.

32. Barraclough TG, Savolainen V: Evolutionary rates and species diversity in flowering plants. Evolution 200I, 55:677-683.

33. Jobson RW, Albert VA: Molecular rates parallel diversification contrasts between carnivorous plant sister lineages. Cladistics 2002, I 8:127-136.

34. Bergthorsson U, Adams KL, Thomason B, Palmer JD: Widespread horizontal transfer of mitochondrial genes in flowering plants. Nature 2003, 424: 197-201.

35. Richardson AO, Palmer JD: Horizontal gene transfer in plants. Exp Bot 2007, 58: I-9.

36. Adams KL, Qiu YL, Stoutemyer M, Palmer JD: Punctuated evolution of mitochondrial gene content: high and variable rates of mitochondrial gene loss and transfer during angiosperm evolution. Proc Natl Acad Sci USA 2002, 99:9905-9912.

37. Perry AS, Wolfe $\mathrm{KH}$ : Nucleotide substitution rates in legume chloroplast DNA depend on the presence of the inverted repeat. I Mol Evol 2002, 55:501-508.
38. Houliston G], Olson MS: Nonneutral evolution of organelle genes in Silene vulgaris. Genetics 2006, I74:1983-1994.

39. Barr CM, Keller SR, Ingvarsson PK, Sloan DB, Taylor DR: Variation in mutation rate and polymorphism among mitochondrial genes of Silene vulgaris. Mol Biol Evol in press.

40. Doyle J], Doyle JL: A rapid DNA isolation procedure for smal quantities of fresh leaf tissues. Phytochem Bull | 987, I 9: | |- I 5.

4I. Qiu YL, Dombrovska O, Lee J, Li L, Whitlock BA, Bernasconi-Quadroni F, Rest JS, Davis CC, Borsch T, Hilu KW, Renner SS, Soltis DE, Soltis PS, Zanis MJ, Cannone JJ, Gutell RR, Powell M, Savolainen V, Chatrou LW, Chase MW: Phylogenetic analyses of basal angiosperms based on nine plastid, mitochondrial, and nuclear genes. Int J Plant Sci 2005, I 66:81 5-842.

42. Stamatakis A: RAxML-VI-HPC: maximum likelihood-based phylogenetic analyses with thousands of taxa and mixed models. Bioinformatics 2006, 22:2688-2690.

43. Kosakovsky Pond SL, Frost SDW, Muse SV: HyPhy: hypothesis testing using phylogenies. Bioinformatics 2005, 2 I :676-679.

44. Sanderson MJ: r8s: inferring absolute rates of molecular evolution and divergence times in the absence of a molecular clock. Bioinformatics 2003, 19:301-302.

45. Wikstrom N, Savolainen V, Chase MW: Evolution of the angiosperms: calibrating the family tree. Proc $R$ Soc London $B$ 200I, 268:22II-2220.
Publish with Biomed Central and every scientist can read your work free of charge

"BioMed Central will be the most significant development for disseminating the results of biomedical research in our lifetime. "

Sir Paul Nurse, Cancer Research UK

Your research papers will be:

- available free of charge to the entire biomedical community

- peer reviewed and published immediately upon acceptance

- cited in PubMed and archived on PubMed Central

- yours - you keep the copyright
BiolMedcentral 